\title{
Towards Consistent-Reliability Structural Design for Earthquakes
}

\author{
Luis Esteva \\ Institute of Engineering, National University of Mexico, \\ Ciudad Universitaria, 04510, Mexico, D. F., Mexico
}

\section{INTRODUCTORY NOTE}

This is an abridged version of Ref. 1. It eliminates most of the references, as well as the discussion concerning the probabilistic modelling of seismic hazard and ground motion, and concentrates on the system reliability concepts.

\section{STATE OF THE ART: AN OVERVIEW}

Establishing reliability-based criteria for earthquake resistant design of structural systems is not an easy task, nor one free of conflicting viewpoints, wide knowledge gaps and significant implementation difficulties. The troubles are linked to the complexities inherent in the phenomena under study --ranging from earthquake generation and propagation to structural response and behavior-- and, of course, to the process of making safety-related decisions.

Take, for instance, the modeling of the seismic excitation. One school of thought advocates the formulation of probabilistic models of the process of earthquake occurrence at a site and of the detailed characteristcs of ground motion time histories during each event. The followers of this approach have to confront the problems arising from the lack of sufficient statistical information on which to base those probabilistic models. Therefore, they have to make simple assumptions concerning the forms of the models, and to estimate the corresponding parameters by means of bayesian statistical analysis. For this purpose, they have to adopt largely subjective prior probability distributions to describe their degrees of belief on sets of alternate assumptions based on geological and geophysical features, as well as on statistical information about events occurred in other seismic regions, considered to be similar to those for which the estimates of activity are to be obtained. The set of mathematical models used to represent the joint probability distributions of earthquake magnitudes and interoccurrence times includes models as simple as the homogeneous Poisson time process with independent random selection of magnitudes and spatial coordinates, and as refined as those that take into account correlation among time, magnitude, spatial coordinates and history of energy liberation by previous activity. 
Opponents to the foregoing approach are found mainly among those responsible for the formulation of design requirements for very important structures. To support their position, they argue against the subjective component of the bayesian estimation approach mentioned above, and against the difficulties associated with extrapolating to the very low hazard interval the time-magnitude-intensity probability distributions derived on the exclusive basis of the direct statistical evidence. They choose, instead, to base their design recommendations on an assumed worst credible event, consisting in the largest earthquake magnitude at the shortest possible distance from the site of interest. This approach is called "deterministic", not because it is devoid of uncertainties, but because the decisions arising from it are supposed to be conservative enough as to make irrelevant any formal evaluation of uncertainty. Although much smaller, uncertainties associated with intensity attenuation, ground motion time history and response spectra for a given magnitude and distance, are covered by the formal probability-based criterion of adopting for design the response values corresponding to a specified number of standard deviations above the mean of the conditional distribution of the maximum response, given the assumed occurrence of the so called worst credible combination of magnitude and source-to-site distance. The rest of this paper will focus on the formal probabilistic approach.

The second link in the seismic reliability analysis chain was mentioned in connection with the deterministic approach (abandoned for the rest of this paper), but it appears as well in the formal probabilistic analysis of seismic hazard. It deals with the use of intensity-attenuation expressions; i.e., algorithms to predict ground motion characteristics for given magnitudes and distances. The first such expressions were used to predict peak amplitudes of ground acceleration, velocity and displacement on firm ground, or ordinates of the response spectra for those local conditions. The information on which they were based included very few records at short hypocentral distances, and therefore their predictions of intensities near the source area are very poor. At moderate distances, typical prediction errors are characterized by variation coefficients of the order of 0.5 .

Our capability for making probabilistic predictions of ground motion intensities and spectral amplitudes has improved substantially during the last few years, mainly as a result of the use of Fourier spectra and random vibration theory, and the introduction of the empirical-Green's function concept. The latter has been particularly valuable for the prediction of high-intensity ground motion characteristics at soft-soil sites. Considerable effort has been devoted to the development of mathematical models and algorithms to study the influence of local soil conditions and some geological and morphological features, but they are not discussed here, as their contribution to the uncertainties about ground motion characteristics will be considered as included in the attenuation expressions specifically applicable to each site of interest.

The study of structural reliability for earthquake actions may be divided into two steps: a) reliability and expected-damage analysis for single earthquakes, of given intensities, and b) integration with respect to the probabilities of occurrence of earthquakes with different characteristics during given time intervals. And there remains the problem of taking into account the influence on structural properties and system reliability of damage accumulation resulting from previous excitations (other earthquakes, differential settlements, etc.). 
Research efforts on the reliability analysis of structural systems subjected to stochastic models of earthquakes have been concentrated on the probabilistic prediction of response, either by means of random vibration theory or by Monte Carlo simulation. Typical results of the former group of methods are the superposition criteria of modal responses of linear multi-degree-of-freedom systems, and the estimation of first passage probabilities of linearsystem responses to segments of stationary and nonstationary gaussian ground motion. Other significant result applicable to the same types of excitation is the development of the method of stochastic equivalent linearization to obtain time-varying covariance matrices of the response vectors of nonlinear hysteretic or deteriorating multi-degree-of-freedom systems with deterministic properties.

Much less attention has been given to the influence of uncertainties about structural properties and to the definition of failure criteria for nonlinear multi-degree-of-freedom systems and to the analysis of failure probabilities of those systems when subjected to earthquakes. References 2 and 3 describe applications of Monte Carlo simulation to evaluate those probabilities for multistory building frames and for simple models of structurefoundation systems with uncertain properties. In order to solve the implied system reliability problem, the set of relevant failure modes is identified, and the maximum value reached of the capacity/demand ratio for any of the failure modes is used as the indicator of system damage. Probability distributions of that ratio are obtained by repeated evaluations of structural responses of systems with Monte Carlo-simulated properties subjected to artificial ground motion records with prescribed statistical properties.

A significant limitation of the few studies that try to evaluate seismic failure probabilities is the arbitrary way in which system failure criteria are defined. However, one of their main conclusions is incontestable: seismic failure probabilities of structural systems designed for the same earthquake spectra and the same general requirements may differ from each other by orders of magnitude. Discussing possible ways to reduce these differences is the main objective of this paper.

\section{GENERAL FRAMEWORK}

For the purpose of developing consistent-reliability seismic design criteria an integrated analysis is required of the concepts described in the following paragraphs (Ref. 1 includes a detailed analysis of these concepts.)

3.1. Seismic hazard: This should be expressed by the joint probability distributions of times of occurrence, intensities and evolutionary spectral functions (ESF), of the earthquakes that may be experienced at a given site.

3.2. System models: These include mass, stiffness, strength and, in general, constitutive laws of structural members subjected to large-alternating strains. The largest contributions to uncertainty about model properties and behavior rules are probably those related with failure criteria and damage indexes. Therefore, they will receive special attention in this paper.

3.3. System-reliability: Having in mind its expected use in the making of safety-related engineering decisions, this concept is best expressed in terms of its complement, that is, a set 
of system failure probabilities for different time intervals or, equivalently, by the probability density function of the waiting time to failure.

3.4. Safety-related decisions: Strategies for the selection of acceptable (or tolerable) failure probabilities for a given system on the basis of trade-off relations among the construction-and maintenance cost increments required to increase safety of that system and the expected reductions in the values of the failure consequences.

3.5. Design formats: The concepts and guidelines presented in the sequel may be formally and explicitly applied by individual designers or groups to very important specific projects. For typical structures to be covered by building codes, however, the best feasible option consists in specifying earthquake design spectra corresponding to different return intervals, to be chosen in accordance with the importance of a structure and the potential consequences of its failure, and a set of rules to determine the safety factors in accordance with the systemreliability characteristics of each structure.

\section{SYSTEM RELIABILITY}

\subsection{Influence of model on dynamic response}

From the discussions above it is concluded that two of the main difficulties for the determination of the seismic reliability of multi-degree-of-freedom structural systems are the possibility of occurrence of several correlated failure modes and the lack of clearly defined mathematical models expressing the conditions required for system-failure.

Another significant difficulty is the high sensitivity of the computed failure probabilities to the types of models adopted to estimate the response of nonlinear systems. For instance, the results presented in Ref. 4 show that the distributions of story ductility demands produced by earthquakes on multi-story buildings are much more irregular for buildings idealized as shear systems than for those represented by continuous, ductile frames. In the former systems, ductility demands are concentrated at the bottom stories, where they attain values several times higher than those appearing at the upper stories of the same buildings or anywhere along the height of the frame-building models.

Ref. 4 also shows that for frames with stiff and strong beams ductility demands may be very sensitive to slight deviations of column strengths as compared with those produced by standard design procedures. This is another argument in favor of the generally recomended strongcolumn-weak-beam design approach.

\subsection{Reliability of elastoplastic systems}

Several single-bay, single-story and multistory frames with uncertain properties were studied in Ref. 2. A set of possible random realizations of each frame was generated by Monte Carlo simulation. The members of each set were then subjected to artificial accelerograms belonging to either of two families: one with the same statistical properties as the El Centro 1940 NS component; the other, similar to the Mexico City 1985 SCT EW component. In order to study the influence of earthquake intensity on the computed failure probabilities, both 
the original and the artificial accelerograms were scaled up and down to several intensities (measured by peak ground acceleration), without changing the time scale.

The determination of system reliability for a given intensity was based on the assumption that under the action of an earthquake, the structure may fail in $\mathbf{n}$ different modes; for instance, each failure mode may correspond to exceedance of the capacity for ductile deformation at a given story. If $S_{i}$ represents the maximum amplitude of the response variable governing the occurrence of the $i$-th failure mode (here, story deformation) and $\mathbf{R}_{\mathbf{i}}$ is the value of $S_{i}$ causing failure, the ratio $Q_{i}=S_{i} / R_{i}$ is the reciprocal of a random safety factor such that $Q_{i} \geq 1$ means failure in the $i$-th mode. It is also assumed that failure occurs precisely in the $i$-th mode and not in any other if $Q_{i} \geq Q_{j}$ for all $j=1, \ldots, n$. This is a simplifying assumption that ignores the possibility that during the response process the condition $Q_{j} \geq 1$ may be reached before the condition $Q_{i} \geq 1$. From these assumptions, the probability of failure for a given intensity $(y)$ equals the probability that the maximum of all the values $Q_{i}$ exceeds unity. Thus, if that maximum is called $Q$, then $P_{r}(y)=P(Q \geq 1$ | y).

Figures 1 to 4 were taken from Ref. 2. In them, $V_{\mu}$ is the variation coefficient of the available story ductility, $\mu$, and $\mu^{*}$ its nominal value, assumed for the purpose of selecting a reduced design spectrum. $\mu^{*}$ and $V_{\mu}$ are related with the mean value $\bar{\mu}$ through the equation $\bar{\mu}=\mu^{\bullet} \exp \left(0.55 \times 3 \times V_{\mu}\right)$. The terms HC and LC designate high and low spatial correlation coefficients. The values of these coefficients as well as of other relevant variables can be found in Ref. 2. Figures 1 and 2 show values of $Q=S / R$ obtained for different earthquake intensities by means of Monte Carlo simulation. They also show empirical expressions for the mean and standard deviation of the natural logarithm of $Q$ in terms of the earthquake intensity, measured by the peak ground acceleration. The curves in figures 3 and 4 were obtained using these expressions, assuming that $Q$ has a lognormal distribution. From these figures, and others similar to them, the following conclusions were reached (2):

1. The number of degrees of freedom has a great influence on the probability of failure.

2. Due to the form of the assumed relation between the expected and the nominal values of the available ductility, as a fuction of the variation coefficient of that variable, the probabilities of failure for a given intensity are greater for those cases for which that variation coefficient is lower.

3. Because the systems considered in the study are assumed to develop significant local yielding at several critical sections before a failure limit state is reached, the results reported are valid only if the safety factors with respect to local brittle failure modes are sufficiently higher than those associated to ductile modes as to prevent the occurrence of the former.

4. The variability of the failure probabilites obtained for the few cases studied justifies the develoment of new studies, with the aim of widening the ranges of the variables covered and exploring the use of better representations of the mechanical behavior of structural members and systems. 


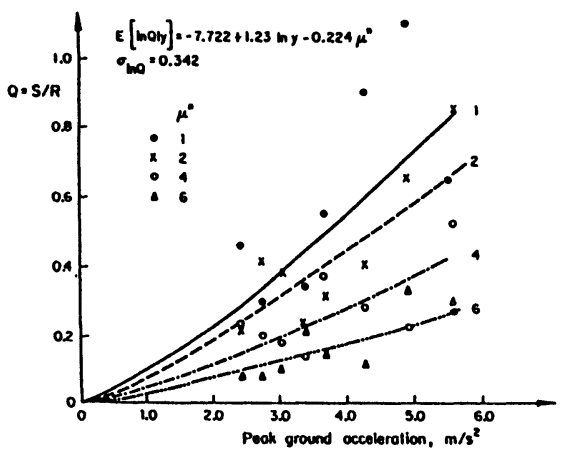

Fig 1 Normolized response of single-slory fromes; $T=0.36 \mathrm{~s}, V_{\mu}=0.3$ (From ref. 2)

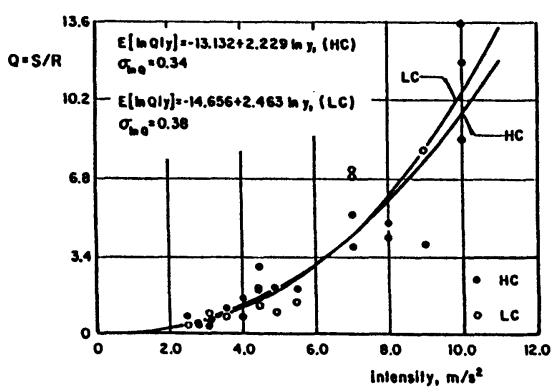

Fig 2 Normalized response of three-story frames; $T=0.36 \mathrm{~s}, \mu^{*}=4, V_{\mu}=0.3$. High $(H C)$ and low (LC) spotial correlation (From ref. 2)

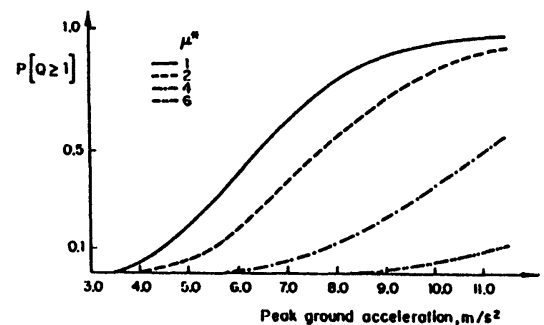

Fig 3 Foilure probobilities of single-story frames designed for different ductility foctors; $v_{\mu}=0.3$ (From ref. 2)

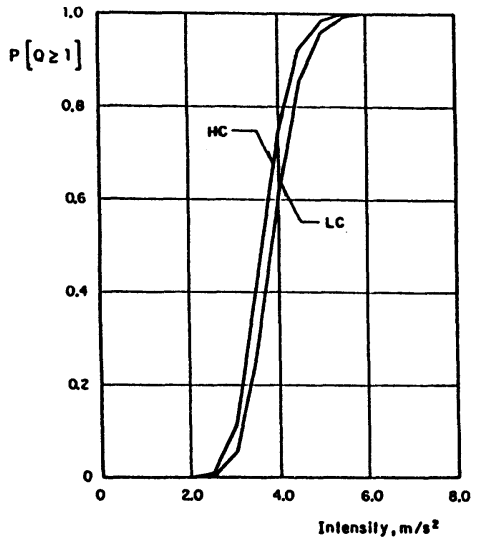

Fig 4 Foilure probobilities of three-story fromes; $T=0.36 \mathrm{~s}, \mu=4, V_{\mu}=0.3$. High (HC) and low (LC) spotial correlation (From ref. 2)

\subsection{Failure rates}

Following Ref. 2, the rate of failure (per unit time) of a structure with uncertain properties (vector $\mathbf{R}$ ) is

$$
\nu_{P}=\int_{0}^{\infty}-\frac{\partial \nu(y)}{\partial y} p_{F}(y) d y
$$

where $\nu(y)$ is the rate of occurrence of earthquakes with intensity greater than $y$ and $p_{p}(y)$ is the failure probability of the structure under the action of that intensity. For the examples described in the foregoing paragraphs, $p_{\mathrm{P}}(y)$ is given by curves as shown in figures 3 and 4.

The resulting failure rates show wide variability with respect to a trend to decrease when the design ductility factors increase. This trend can be explained because the higher the capacity of a structure to resist ductile deformations, the lower the lateral strength (additional to that available in a continuous frame designed for gravitational loads) required to resist a specified set of lateral forces and, therefore, the higher in proportion is the contribution of the member resistances needed for vertical loads to the lateral strength required to take an earthquake of a given intensity. 
The wide variability of the normalized failure rates reflects that shown by the failure probability functions similar to those shown in Figs 3 and 4. This variability shows how far present design criteria are from our consistent-reliability goal. As important as to accomplish consistency among computed failure probabilities it is to improve the criteria used to define system failure and to calibrate response, damage and failure models with the observed behavior of actual systems.

\subsection{Improved response models and failure criteria}

One possible way of defining the failure conditions in multistory buildings subjected to earthquake ground motion is to express those conditions in terms of the damage accumulated throughout the members which participate in each failure mechanism. For instance, for failure associated with excessive damage accumulation at the ends of the columns and beams of a given story, damage can be measured by a sum of terms proportional to $x_{i}^{4}$, where $x_{i}$ is the absolute value of the story deformation amplitude during the $i$-th cycle of its response time-history. Still, it is not easy to establish the value of the accumulated damage at which the story fails. A more practical indicator of damage, strongly correlated with the formerly proposed measure, is the decrease in the story-stiffness. If failure is expressed by the condition that the secant story stiffness $\left(\mathrm{K}_{2}\right)$ corresponding to the maximum story deformation during a given cycle of the response time history is equal to zero, then the accumulated damage can be measured by $\delta=\left(\mathrm{K}_{\mathrm{o}}-\mathrm{K}_{\mathrm{m}}\right) / \mathrm{K}_{0}$, where $\mathrm{K}_{0}$ is the small-deformation tangent initial stiffness for the undamaged structure, and $K_{m}$ is the minimum value of $K_{\text {, }}$.

The damage accumulation and the associated stiffness reduction for any given story will be determined by the corresponding local damage processes at the ends of the structural members of the story of interest and of those adjacent to it. Important efforts have been made during the last few years to develop realistic stiffness- and strength-degrading models of the momentcurvature (or moment-rotation) constitutive laws of the plastic hinges when subjected to repeated load applications $(5,6)$. The model proposed in Ref. 6 has been applied in introductory studies about the influence of cumulative damage on the seismic reliability of multistory frames and about the formulation of optimum decisions related to design and replacement of seismic energy dissipators. Those applications brought to the surface both the limitations of the model and the sensitivity of the computed failure probabilities to its form and parameters. However, the writer considers that the use of damaged-based stiffnessdegrading models (DBSD, models relating stiffness degrading with accumulated damage) constitute a promising possibility for the modeling of system failure in the study of seismic structural reliability. This has stimulated the development of a new DBSD model, inspired on that of Ref. 6, and described in the sequel.

The model proposed is presented in Fig. 5. The upper portion shows an idealized momentrotation behavior at one end of a simply supported bending member, with the other end free to rotate. Following the model adopted --linear elastic member with plastic hinges at the ends-- the tangent initial stiffness is that of the linear elastic member, while the deformations in excess of those represented by line $0^{\circ}-0^{\circ}$ are the rotations concentrated at the plastic hinge. The behavior of the latter is shown in the lower portion of the figure. Damage accumulation is assumed to take place independently in each of the two opposite loading directions. In each direction, damage is measured by the sum of the plastic deformations in that direction divided by the yield deformation of the virgin curve. The algorithm which transforms accumulated 
damage into stiffness reduction can be better explained following the cyclic response curves in Fig. 5. Suppose that when point b in Figs $5 a$ and $b$ is reached, the damage already accumulated in the positive direction of $\theta$ is $D_{b}$. A consequence of this damage is that the next reloading cycle in the positive direction of the $\theta_{\mathrm{p}}$ axis in Fig. $5 \mathrm{~b}$ will be directed from point $f$ to point $b_{1}$, and may eventually reach the envelope curve at point $g$. If $y_{b}$ and $y_{b 1}$ are respectively the ordinates of points $b$ and $b_{1}$, corresponding to the moments acting at the beam end for a plastic hinge rotation equal to $\theta_{\mathrm{pb}}$ (segment $\alpha c$ in Fig. $5 b$ ), then the level of degradation is given by the ratio $\varepsilon_{b}=\frac{y_{b}-y_{b l}}{y_{b}}$. This ratio is a function of the damage $\mathrm{D}_{b}$ accumulated up to point b: $\varepsilon_{b}=\varepsilon\left(D_{b}\right)$. A similar relation exists between $\varepsilon_{h}=\frac{y_{h}-y_{h l}}{y_{h}}$ and $\mathrm{D}_{\mathrm{h}}$, and between $\varepsilon_{m}=\frac{y_{m}-y_{m l}}{y_{m}}$ and $\mathrm{D}_{m}$. As figures 5a and $\mathrm{b}$ also show, the reloading branches may be directed towards a point in segment $\mathrm{AB}$ of the envelope, or may have such a small stiffness that they or their projections intersect the envelope at a point in segment BC.
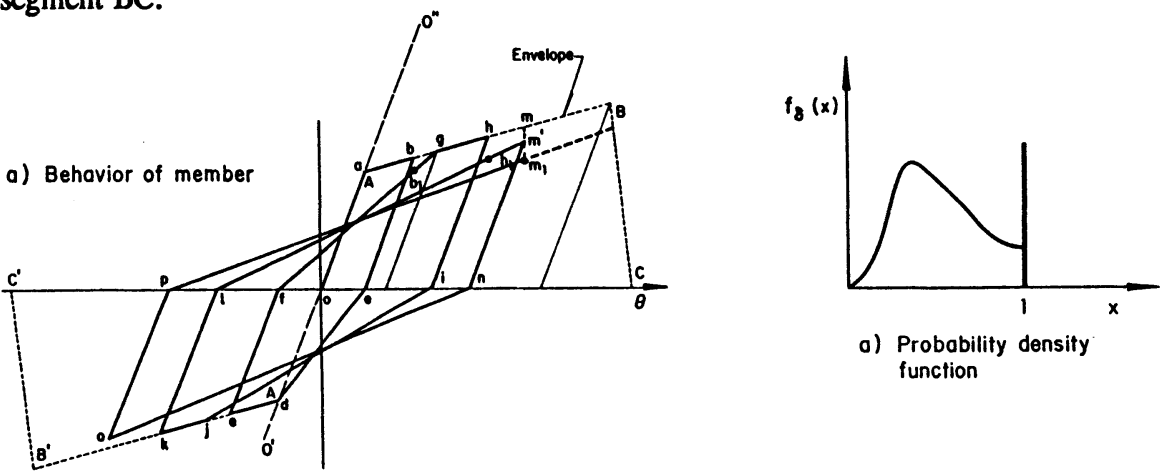

a) Probability density function
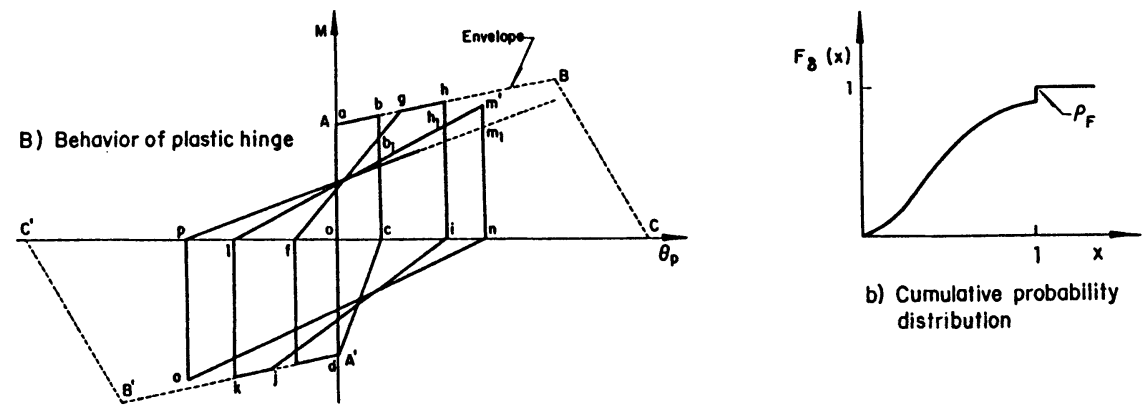

b) Cumulative probability distributlon

Fig 5 Stiffness and strength-degrading models of bending member and plastic hinge

Fig 6 Probability distribution of $\delta_{\max }$ 


\subsection{A new approach to seismic reliability analysis}

It is not easy to compute an indicator similar to D for a given story or subassemblage of a structure when obtaining the time-history of its dynamic response to a ground motion accelerogram, but it is reasonably simple to obtain values of $\mathrm{K}$, of any story throughout the time when the response of the system remains sufficiently high as to be significant for engineering purposes. This statement can be extended to the stiffnesses related to failure mechanisms other than excessive story deformation. Thus, the problem of determining the probability of failure of a system under the action of earthquakes randomly selected from a given population (which, of course, includes the case of earthquakes with a given intensity) is transformed into one of a) identifying a set of potential failure modes, each associated to the occurrence of excessive deformations of a certain portion or subassemblage of the system; b) expressing the response in each mode in terms of an effective force and an effective deformation, such that failure in that mode takes place when the value of $K_{1}$ expressed in terms of them becomes null; and c) obtaining the probability that any of the $K_{\mathbf{m}}$ becomes null.

If $\delta_{i}, i=1, N_{\mathbf{m}}$ is the ratio $\left(\mathrm{K}_{\mathrm{oi}}-\mathrm{K}_{\mathrm{min}}\right) / \mathrm{K}_{\mathrm{oi}}$ for the $i$-th mode and $\mathrm{N}_{\mathbf{m}}$ is the number of potential failure modes, then the latter probability is equal to that of the event that any of the $\delta^{\prime}, s$ reaches a value of 1 , or of the equivalent event that the maximum $\delta_{t}$, called here $\delta_{\max }$, equals 1 . The probability density function of $\delta_{\operatorname{mx}}$ has a shape similar to Fig. 6a. The spike at 1 has an area equal to the failure probability, equal to the discontinuity shown by the cumulative distribution depicted in Fig. $6 \mathrm{~b}$. If the value of this probability is low, the number of response analyses required to estimate it by counting the number of failures predicted by Monte Carlo simulation will be large. However, the information about values of $\delta_{\max }$ smaller than 1 obtained by Monte Carlo simulation is also significant for the estimation of $\mathrm{p}_{\mathrm{p}}$.

If the form of $f_{\max }(x)$ for $x<1$ is assumed known except for a set $\{\alpha\}$ of undetermined parameters, the estimation of these parameters may be formulated, for instance, by a maximum likelihood criterion or, more generally, resorting to bayesian statistical analysis. Regardless of which of these approaches is adopted, the solution rests on the calculation of the likelihood function for a sample consisting of $n$ values of $\delta_{\max }$ smaller than $1\left(x_{1}, i=1\right.$, n) and $n_{1}$ values equal to 1 . Formally, let

$$
f_{z_{-}}(x)=f(x)+p_{F} \delta(x-1)
$$

where $\delta($.$) is Dirac's delta function and the integral of f(x)$ between the extremes 0 and 1 equals $1-p_{\mathrm{p}}$. This means that, if $f(x)$ is chosen as a probability density function such that its integral from zero to infinity equals $1, p_{p}$ is equal to the integral of $f(x)$ from 1 to infinity; therefore, it is a function of $\{\alpha\}$. Given the sample described above, the likelihood function is

$$
L\left(\alpha \mid x_{i}, i=1, n ; n_{1}\right)=(1-F(1 \mid \alpha))^{n_{1}} \prod_{i=1}^{n} f\left(x_{i} \mid \alpha\right)
$$

where $F(x)=\int_{0}^{x} f(u) d u$. Passing from here to maximum likelihood estimates or bayesian analysis is straightforward. 
A drawback of the foregoing approach is that the results may depend excessively on the choice of $f(x)$. This may be a valid argument when the interest is centered on the study of failure probabilities for given intensities, and those probabilities are very low. However, in most earthquake engineering problems the most important contribution to the uncertainty about the ratio of excitation to capacity comes from the uncertainty about the ground motion intensity. For those cases, the sensitivity of marginal failure probabilities to the form of $f(x)$ is not so pronounced. But the study of this problem must accompany the implementation of the proposed methodology.

Finally, this approach opens the door to the possibility of studying the influence of cummulative damage on the reliability of structural systems to sequences of earthquakes (7).

\subsection{Model uncertainty}

The constitutive laws adopted to represent the behavior of structural members are in general simplified idealizations of complex, imperfectly known phenomena. They are based on very limited experimental information, most of it obtained under laboratory conditions, and therefore requiring extrapolation to natural conditions. Predicted responses to high-intensity earthquakes are very sensitive to model uncertainties --both model form and model parameters-- and so are the probabilistic predictions of response and the reliability estimates derived from them. Accounting for these uncertainties and reducing them through calibration of theoretical models with empirical evidence are key links in the excitation-responsereliability chain.

Calibrating structural-response and system-reliability models for high earthquake intensities implies contrasting observations of response and behavior with the predictions resulting from alternate model forms or parameters. Available field observations come in most cases from low- or moderate-intensity earthquakes, and they must be complemented with laboratory simulations of responses to high-intensity events. The nature of the information that can be obtained varies widely. Laboratory tests in most cases can only be performed on simple structures, but accurate instrumental and descriptive information can be obtained covering both general and local response functions and failure mechanisms; they can also provide qualitative (fuzzy) information about damage levels. Field observations can be made on complex systems, but the degree of complexity significantly affects our capability for interpreting the records. Instrumental information usually refers to global response time histories, but with adequate planning it can also include time histories of deformations strongly correlated with safety margins for specific failure mechanisms or modes. Another valuable outcome of these observation is, of course, the qualitative information about damage severity and space distribution.

Whatever the origin of the empirical information to be used in the calibration of mathematical models, the process includes evaluation and adjustment of the capacity to predict a) structural response, at various levels of detail (global, sub-assemblage, local); b) variation of the properties of simple, equivalent systems; and c) damage severity and distribution. Statistical information about numbers of failures of structures of given types and designed in accordance with given design criteria and quality control norms also provides useful calibration points, but due to the (fortunate) usual shortness of these numbers, our calibrations of failure-probability prediction models will rely more heavily on the information furnished 
by moderate numbers of severely damaged (but not collapsed) structures.

Among the challenges to be found in calibration studies, the following deserve special mention:

a) Identifying critical failure modes and deploying instrument arrays aimed at providing information about the corresponding safety margins.

b) Developing criteria and methods to relate information about global system response with safety margins for specific failure modes.

c) Relating moderate-intensity calibration results with those that should apply for higher intensities.

In spite of their limitations as predictors of system response and behavior, computer methods of response analysis can be used to develop probabilistic information about the relations among global, subassemblage and local responses, thus permitting the application of the methods proposed in point b) above. The suggested transformation can be achieved by means of Bayes' theorem, as explained in the following. Let $\mathrm{X}$ be the vector of responses of interest, which are used in the estimations of safety margins for specific failure modes. (In a multistory building, vector $X$ would contain maximum instantaneous values of lateral story deformations or minimum instantaneous values of secant story stiffnesses.) Let $Z$ be a vector of measured values of some elements of the (global) response time history. Suppose that, for a random ground motion with intensity and frequency content similar to those of the earthquake for which $\mathrm{Z}$ was measured, our knowledge about the model parameters is expressed by the a priori bayesian probability density function of the parameters of a probabilistic model of known form. (Accounting for the uncertainty about model form can easily be incorporated, at least in theory.) Let $\alpha$ be the vector of the mentioned parameters and $\mathrm{f}_{\alpha}{ }^{\prime}($.$) its a priori probability density function. Application of Bayes' theorem leads to$ the a posteriori probability density function:

$$
f_{\alpha}^{\prime \prime}(u \mid z) \propto f_{\alpha}^{\prime}(u) p(z \mid u)
$$

where $\mathrm{p}(\mathrm{z} \mid \mathrm{u})$ is the likelihood function of $\alpha$ corresponding to the observation $\mathrm{Z}=\mathrm{z}$. The $a$ posteriori probability density function of $\mathrm{X}$ is obtained by integrating with respect to the $a$ posteriori probability density function of $\alpha$ :

$$
f_{x}^{\prime \prime}(x \mid z)=\int f_{x}(x ; u) f_{\alpha}^{\prime \prime}(u \mid z) d u
$$

Of course, the value of the information on $\mathrm{Z}$ to improve our knowledge about $\alpha$ would depend on the sensitivity of $\mathrm{Z}$ to $\alpha$. This should be a basic consideration at the stage of planning the location of the instruments on the systems used for calibration. 


\section{CONSISTENT RELIABUITY DESIGN CRITERIA}

The foregoing sections deal with uncertainty analysis and synthesis for the purpose of computing failure probabilities; they consider both single earthquake events and sequences that may occur during given time intervals. The rest of the paper deals with the selection of target reliability levels, and with the definition of structural design formats and values required to attain those levels.

\subsection{Iso-reliability spectrum for given intensity and frequency content}

This constitutes a simplified approach to the situation when the design requirements aim at a specified reliability for an earthquake intensity corresponding to a given return interval at a given site, or for the most unfavorable excitation that may affect that site. It is presented here to illustrate some features of the more general case when the intended reliability level refers to the worst condition that may affect the structure of interest during a given time interval.

Both the intensity and the evolutionary frequency content are assumed given, leaving the detailed ground motion time-history as random. The structures studied in this example are elasto-plastic single-degree-of-freedom systems with uncertain mass, stiffness, lateral strength, and failure deformation, the latter expressed as the product of an "available" ductility factor by the yield deformation. Each system analyzed was defined in terms of the mean values and coefficients of variation of the properties mentioned above. For each of them a nominal natural vibration period was defined, as that computed in terms of the mean values of mass and stiffness. The values assumed for this period range between $0.25 \mathrm{~s}$ and $5 \mathrm{~s}$. The mean and the nominal values $\left(\bar{\mu}, \mu^{*}\right.$, respectively) of the available ductility $(\mu$, with a variation coefficient $\left.V_{\mu}\right)$, are related through the equation $\mu^{*}=\bar{\mu} \exp \left(-2 V_{\mu}\right)$. Several alternative values were assumed for the mean lateral strength. These were sequentially chosen during the study, with the aim of covering an interval of values of the safety index $\beta$ comprised between 1.0 and 6.0. The following variation coefficients were assumed: mass, 0.08 ; stiffness, 0.10 ; available ductility, 0.25 ; lateral strength, 0.15 and 0.30 . A correlation coefficient of 0.7 was assumed between strength and stiffness; the other variables were taken as stochastically independent.

For the application of Monte Carlo method, several samples of values of mass, stiffness, strength and available ductility were generated for each possible combination among the mean values and variation coefficients of these variables. Each sample determined a system which was subjected to several artificial accelerograms similar in intensity and frequency content to the EW component of the Mexico City SCT accelerogram of 19 September 1985 . The simulated accelerograms were normalized to the same values of Arias' intensity. The maximum ductility demand of each system for each accelerogram was obtained from its calculated dynamic response time history. A safety factor was calculated for each case analyzed, as the ratio of available ductility to ductility demand. The value of the safety index $\beta$ was calculated for each combination of structural properties, as the ratio of the mean to the standard deviation of the safety factor. Typical results are shown in Figs. 7-10, corresponding respectively to nominal design ductilities, $\mu^{*}$, of 4 and 2 , and variation coefficients of lateral strength, $V_{R}$, of 0.15 and 0.30 . The ordinates, expressed as spectral accelerations, correspond to expected values of lateral strength leading to the specified $\beta$ 
values for the family of accelerograms considered. The average linear spectral ordinates for 0.05 damping for this family are also shown in the figures, for comparison with the isoreliability spectra. It is clearly seen that the latter may adopt forms which differ substantially from those corresponding to the average linear spectra, and even from those adopted by nonlinear systems with deterministically known properties. The deviations are very sensitive to the uncertainty associated to the natural period of the structure, at least for the narrow-band ground motion accelerograms considered in the study reported.

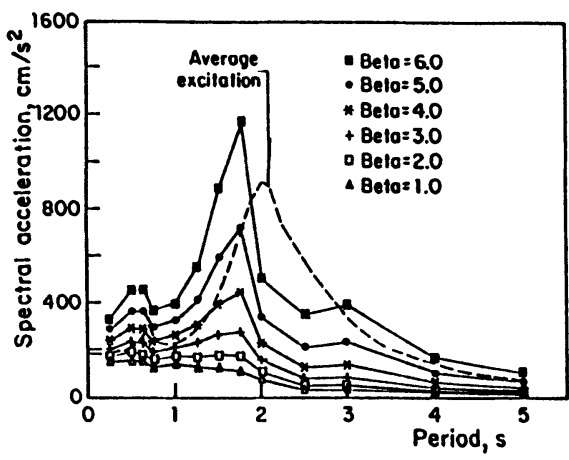

Fig 7 Spectral ordinates for given $\beta_{;} \mu^{*}=4$, $V_{R}=0.15$

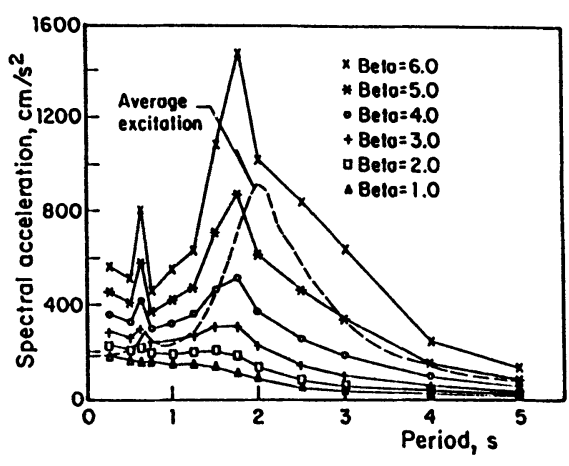

Fig 8 Spectral ordinates for given $\beta ; \mu^{*}=4$, $V_{R}=0.30$

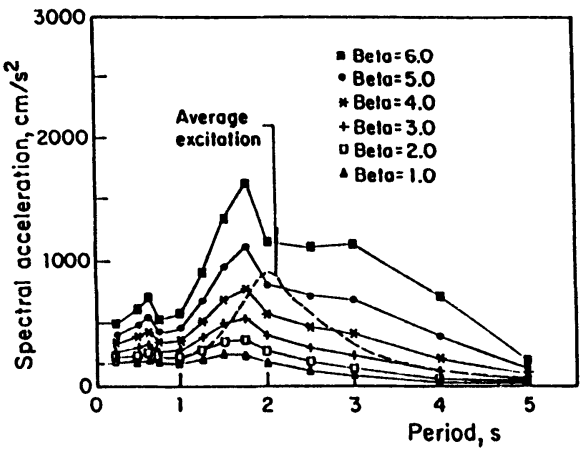

Fig 9 Spectral ordinates for given $\beta ; \mu^{*}=2$, $V_{R}=0.15$

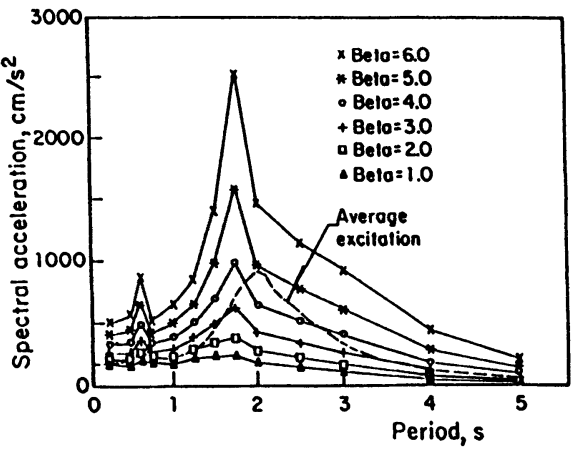

Fig 10 Spectral ordinates for given $\beta ; \mu^{*}=2$, $V_{R}=0.30$

The differences in required strength that may arise from alternate assumptions regarding uncertainties about mass, stiffness, strength and deformation capacity have not been systematically studied, even at an exploratory level. The wide variabilities which characterize those uncertainties in practical cases point at the convenience of undertaking deeper and more extensive studies on this topic, including multi-degree-of-freedom systems. 
Finally, when the determination of the target safety level is formulated as an optimization problem, it may be convenient to refer its value to an expected failure rate per unit time, rather than to a failure probability for an earthquake with specified intensity and frequency content. The forms of the corresponding iso-reliability design spectra do not necessarily coincide with those of the cases studied above.

\subsection{Design format}

From the above it is concluded that conventional earthquake-resistant design for specified response spectra does not lead to consistent reliability levels for structures of different types, and not even of the same type but with different numbers of degrees of freedom. It is clear that we have to learn much about failure probabilities of different systems and understand the influence of uncertainties about several variables before we can significantly improve the consistency of codified design rules. And we will also have to transform our findings on easy-to-apply recommendations. It is very likely that the best option will include the following concepts.

1. Several sets of nonlinear response spectra for structures with different energydissipation characteristics. Each set should correspond to a given return interval (or to its reciprocal, an exceedance rate), to be selected in accordance with the importance of the system to be designed and/or the expected consequences of its failure. These spectra should lead to specified failure rates for single-degree-of-freedom systems with typical uncertainties about their mechanical properties.

2. Load factors, expressed as functions of the nominal natural period of vibration, for systems of different types. These load factor functions should take care of all relevant influences on system reliability.

The determination of load factor functions can be accomplished according to the following steps:

1. Response spectra for given exceedance rates are determined. The exceedance rates selected should be of the order of twenty times the target failure rates.

2. Typical structures belonging to each family being studied are designed for the spectra defined in step 1.

3. Failure probability functions in terms of intensity $\left(p_{F}(y)\right)$ are obtained in accordance with criteria discussed in previous sections.

4. Expected failure rates $\nu_{\mathrm{P}}$ are obtained by combining the failure probability functions $\mathrm{p}_{\mathrm{p}}(\mathrm{y})$ with the intensity-recurrence functions $v(\mathrm{y})$.

5. The cycle is repeated until the calculated $\nu_{\mathrm{F}}$ values are sufficiently close to those aimed for.

The size of the effort required is not small, but the benefits expected from attaining consistent reliability levels justify it. 


\subsection{Consistent-safety decisions}

Structural reliabilities for different structures need not (indeed, must not) be equal, nor do they for similar structures at different locations. They should be optimum, in the sense of maximizing a utility function consisting of a sum of positive (benefits) and negative terms (initial and maintenance costs, damage and failure costs and consequences). Because these contributions are uncertain, their values must be expressed as the expectations of the mentioned variables. Because they may occur at different instants in the future, we must work with their present values; that is, we must add up the expected values of the positive or negative contributions that may take place in the future, each multiplied by an actualization factor, which we will take as $\exp (-\gamma t)$, where $t$ is the time of its occurrence and $\gamma$ is an effective discount rate.

The difficulties that hinder formal applications of the optimization criterion sketched above are well recognized. Prominent among the causes for those limitations are some of philosophical nature: how to assign values to non-monetary losses, such as human deaths or injuries, social disruption, political instability, etc. The analysis of these problems constitutes a focus of interdisciplinary research of engineers and social scientists and is out the scope of this paper. Other difficulties arise from the embrionic stage of development of the methods for computing seismic failure probabilities, and have been dicussed above. Therefore, most of the usefulness of the formal optimization process advocated in this section does not lie at present on its direct application to obtaining optimum design spectra for specific systems but mainly on its use as a calibration tool for the formulation of mutually consistent sets of seismic design spectra for given familis of structures (8).

\section{FINAL COMMENTS}

The advances of the last few decades in the areas of seismic hazard analysis and random vibration theory have permitted the development of practical criteria and methods applicable to some concepts of great importance in the seismic reliability analysis of engineering systems and, consequently, in the corresponding structural design methods. The estimation of intensity-recurrence functions and the application of random vibration analysis to approximately represent the ground motion or to make probabilistic predictions of the responses of linear and (some) nonlinear systems are among the most important results. However, very little has been done to obtain estimates of the failure probabilities of complex nonlinear systems, closely representing real systems, under the action of random earthquakes and sequences of earthquakes, and to assess the validity of those estimates in the light of observations during moderate- or high-intensity earthquakes. Consequently, very little profit has been made of the progress in probabilistic response analysis methods to attain consistent, optimum reliability levels for structures of different types. This explains the interest of the writer about the needs, rather than about the accomplishments.

The intention of this paper has been to provide an integrated view of the problems of singleearthquake and long-term reliabilities of complex systems, of the decisions required to set the target safety levels, of the research required in the system-reliability-area, and of possible formats for implementing the knowledge to be developed into codified design rules. It also intends to contribute to the solution of some of the individual problems pointed at; in 
particular, of the analysis of seismic system-reliability. The efforts related with systemmodeling are concentrated on building frames. This is just for illustrative purposes, but it is recognized that the modeling of other typical structures may be more complicated and is less developed. Also, damage other than that due to system collapse was not discussed, despite its importance in design criteria related with serviceability failure modes. These concepts should receive more attention in the near future.

Finally, several very important topics are either ignored or only cursorily mentioned in this paper: uncertainties about seismic hazard models and parameters, social consequences of catastrophic failures, and risk-related social attitudes, to name a few. The challenges we have to face are huge, but the benefits we may expect are worth the efforts.

\section{REFERENCES}

1. Esteva, L., "Towards consistent-reliability structural design for earthquakes", manuscrip prepared for a keynote lecture presented at the 6th IFIP WG7.5 Working Conference, Assissi, Italy (1994).

2. Esteva. L., and Ruiz, S. E., "Seismic failure rates of multistory frames", ASCE Journal of Structural Engineering, 115, 2 (1989), 268-284.

3. Esteva, L., Díaz, O., Mendoza, E., and Quiroz, N., "Reliability based design requirements for foundations of buildings subjected to earthquake", Proc. ICOSSAR 89, San Francisco (1989).

4. Díaz, O., Mendoza, E., and Esteva, L., "Seismic ductility demands predicted by alternate models of building frames", Earthquake Spectra, 10, 3 (1994), 465-488.

5. Park, Y. J., Reinhorn, A.M. and Kunnath, S.K., "IDARC": Inelastic Damage Analysis of Reinforced Concrete Frame-shear-wall Structures", Technical Report NCEER-87-0008, State University of New York at Buffalo, July 1987.

6. Shah, S. P., and Wang, M. L., "Reinforced concrete hysteresis model based on the damage concept", Earthquake Engineering and Structural Dynamics, 15 (1987), 993-1003.

7. Esteva, L., and Díaz, O., "Optimum decisions related to design and replacement of seismic energy dissipators", Structural Safety and Reliability, Proc. of ICOSSAR 93, Balkema, Rotterdam (1993), 653-660.

8. Esteva, L., and Ordaz, M., "Riesgo sísmico y espectros de diseño en la República Mexicana", accepted for publication in Revista de la Sociedad Mexicana de Ingeniería Sísmica (1994). 